\title{
Magnetic-Field-Induced Spin-Conserving and Spin-Flip Intersubband Transitions in InAs Quantum Wells
}

\author{
C. Gauer, A. Wixforth, and J.P. Kotthaus \\ Sektion Physik der Ludwig-Maximilians-Universität München, D-80539 München, Germany \\ M. Kubisa \\ Institute of Physics, Polish Academy of Science, 02668 Warsaw, Poland \\ W. Zawadzki* \\ Institute for Experimental Physics, University of Innsbruck, A-6020 Innsbruck, Austria \\ B. Brar and H. Kroemer \\ Department of Electrical and Computer Engineering, The University of California at Santa Barbara, Santa Barbara, \\ California 93106 \\ (Received 2 November 1994)
}

\begin{abstract}
Intersubband optical resonances in InAs/AlSb quantum wells have been studied in the Voigt configuration. Both spin-conserving and spin-flip transitions in the midinfrared spectral range were observed simultaneously. We demonstrate that the in-plane magnetic field mediates the spin-conserving transitions, whereas the bulk inversion asymmetry of InAs induces spin-flip excitations. The difference in the observed resonance energies allows us to deduce directly the depolarization shift. Moreover, we show that this depolarization strongly reduces the linewidth of the spin-conserving resonance.
\end{abstract}

PACS numbers: 71.70.Ej, 73.20.At, 73.20.Dx

Many-body effects in two-dimensional electron gases (2DEGs) have been the subject of detailed investigations during the last few years. Especially inelastic light scattering has been proven to be a powerful method because charge- and spin-density excitations can be measured simultaneously [1]. So far, in absorption spectroscopy only combined cyclotron intersubband resonances [2,3] provide direct information on many-body effects. This is because in contrast to inelastic light scattering the dipole selection rules for intersubband resonance require a strong spin-orbit interaction for the observation of spin excitations. Thus attention has focused on either hole systems [4] or electrons in narrow-gap materials, in which interband coupling leads to strong spin-orbit interaction [5]. For example, electric-dipole-excited spin resonances in bulk InSb have contributed greatly to the understanding of the band structure and particularly the role of bulk inversion asymmetry as present in III-V compounds [6]. The latter not only enhances spin-flip transitions but also leads to a zero-field spin splitting at finite $k_{\|}$also for a 2DEG [7].

Here, we report on the experimental and theoretical investigations of optical transitions between hybrid magnetoelectric subbands (HMES) [8] of InAs/AlSb multiple quantum wells. This new material combination provides high mobility 2DEGs with strong spin-orbit interaction which makes it a promising system to observe spin excitations. Our experimental approach combines the specific advantages of inelastic light scattering and absorption spectroscopy as both spin-conserving and spin-flip transitions can be observed simultaneously. Usually, inter- subband transitions (IST) are only allowed for light polarization in the direction of the electric confinement [9] unless the parallel and perpendicular motion are strongly coupled as, for example, in tilted valley systems [10]. We show that an in-plane magnetic field breaks this polarization selection rule and that the inherent bulk inversion asymmetry of our quantum well material gives rise to an excitation mechanism for spin-flip IST. Futhermore, we can simply deduce the depolarization shift of the spinconserving transition from its energetic difference to the spin-flip IST. Comparing the linewidths of the spin-flip and the spin-conserving resonances we find that the depolarization drastically reduces the line broadening of the latter.

The sample described here was grown on a GaAs substrate followed by a sequence of buffer layers [11] to accommodate the relatively large lattice mismatch between the substrate and the active layers. The 20 period multiple quantum well consists of $15 \mathrm{~nm}$ InAs layers and $10 \mathrm{~nm}$ wide $\delta$-doped Te:AlSb barriers. To minimize band-bending effects [12] we chose a $55 \mathrm{~nm}$ thick topmost AlSb barrier capped by a $5 \mathrm{~nm}$ wide layer of GaSb. Hall effect measurements at low temperatures revealed a carrier density of $N_{s}=2.5 \times 10^{12} \mathrm{~cm}^{-2}$ per well. The mid-infrared spectra were taken using a rapidscan Fourier transform spectrometer with the samples mounted in Voigt geometry in the center of a superconducting solenoid. Experimentally, we determined the relative change in the transmission $T(B \neq 0) / T(B=0)$ under normal incidence of unpolarized light for different in-plane magnetic fields $B$. Alternately, we 
could ratio the spectra against those of a reference substrate.

In Fig. 1 we show a transmission spectrum of our sample taken under an oblique angle of $57^{\circ}$ used in a ratio against a reference substrate (upper trace). In this conventional IST configuration the refraction of the incident light results in a finite electric field component perpendicular to the $2 \mathrm{DEG}$ as required to excite an IST at $B=0 \mathrm{~T}$. The extracted resonance position agrees quite well with the one theoretically expected. Also the oscillator strength is found to exhibit the calculated dependence on the tilt angle (not shown in the figure).

In the Voigt configuration, i.e., the light incident perpendicular to the sample plane and the in-plane magnetic field, we obtain the lower traces of Fig. 1. The spectra have been taken between $B=2 \mathrm{~T}$ and $B=13 \mathrm{~T}$ with a reference at $B=0$. Surprisingly, we observe two well separated resonances - one at around $E=100 \mathrm{meV}(A)$ and a second at $E=135 \mathrm{meV}(C)$. In an additional experiment which is not shown here we verified that for the in-plane light polarization no absorption is detectable at

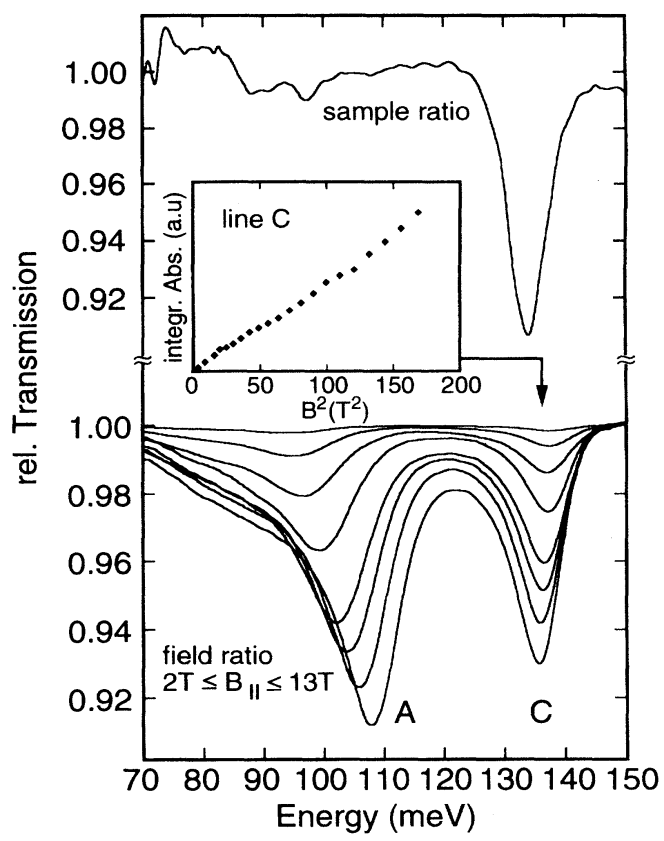

FIG. 1. The upper trace shows a transmission spectrum of our InAs/AlSb multiple quantum well used in a ratio against a reference substrate with the radiation incident under an oblique angle of $57^{\circ}$. The strength of the resonance follows the same polarization dependence as expected for intersubband transitions. We are not yet certain how to interpret the small structures around $E=90 \mathrm{meV}$ as they may be caused by the ratio. In the magnetic field ratio of the same sample we show spectra $T(B \neq 0) / T(B=0)$ at in-plane magnetic fields $B=2$, $4,6,8,10,11,12$, and $13 \mathrm{~T}$. Both resonances $A$ and $C$ vanish at zero magnetic field, and their absorption increases at higher fields. The inset shows that the integrated absorption of line $C$ depends quadratically on the magnetic field. zero magnetic field. Hence, we conclude that both resonances $A$ and $C$ are induced by the magnetic field and gain strength at high fields. With increasing field, the position of line $A$ shifts to higher energies from $E=94 \mathrm{meV}$ at $B=2 \mathrm{~T}$ to $E=109 \mathrm{meV}$ at $B=13 \mathrm{~T}$, whereas the position of the much narrower line $C$ remains almost constant. Furthermore, line $C$ is symmetric, and its width does not depend on the magnetic field. Its integrated absorption, however, increases quadratically with the field (inset of Fig. 1). Comparing with the top trace, resonance $C$ is readily identified as the depolarization shifted IST. This conclusion is based on the fact that the energetic position and line shape are almost identical in both spectra. The low-energy resonance $A$, on the other hand, gains oscillator strength by an increase of both the absorption depth and the linewidth. It is considerably broader than line $C$ at all fields and also becomes more and more asymmetric as the magnetic field is increased.

To understand the spectra in more detail we calculate the single particle subband energies within the framework of a three-level $\vec{k} \cdot \vec{p}$ model [13] as a function of the parallel magnetic field, where bending of the quantum well bottom has been taken into account by first order perturbation theory. Throughout this Letter we choose the direction of growth and the electric confinement to be $y$ while the magnetic field is applied in the $z$ direction $\vec{B} \| z$. In Fig. 2 we plot the intersubband transition energies $\Delta E$ for $k_{x}=k_{z}=0$ and in the inset a schematic of

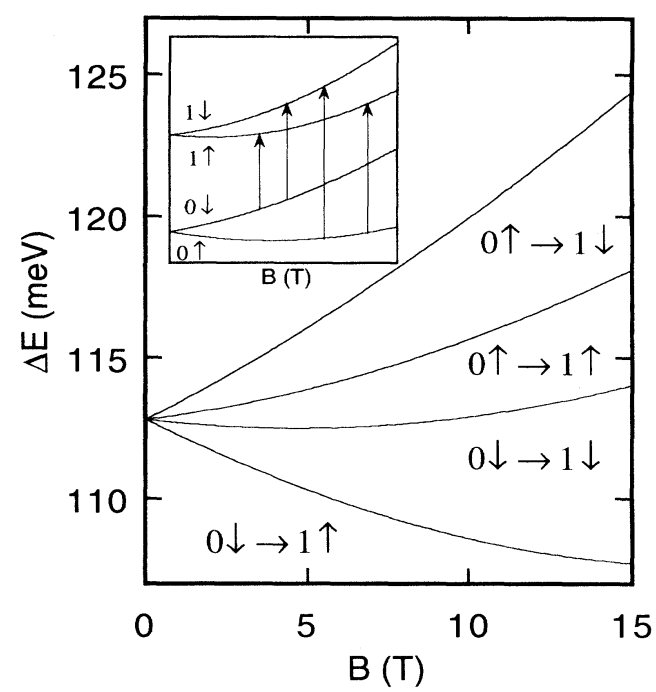

FIG. 2. Single particle transition energies between the HMES for $k_{x}=k_{z}=0$. The spin-conserving transitions differ only very little in energy while the spin-flip excitations are energetically more separated. As the band parameters were take $E_{g}=0.42 \mathrm{eV}, \Delta=0.38 \mathrm{eV}$, and the Kane energy $E_{p}=$ $22.9 \mathrm{eV}$. The inset shows schematically the relevant subband edges as a function of the magnetic field. The spin-up branch is lower in energy than the spin-down branch because the electronic $g$ factor of InAs is negative. 
the relevant subband edges as a function of magnetic field. From the four possible intersubband excitations two are spin conserving, while the two others involve a spin flip. The transition energies are almost identical for $|0 \uparrow\rangle \rightarrow|1 \uparrow\rangle$ and $|0 \downarrow\rangle \rightarrow|1 \downarrow\rangle$, and they shift only slightly at high magnetic fields. The spin-flip transition energies, on the other hand, are more widely separated. At $B=$ $13 \mathrm{~T}$ the energetic difference between $|0 \uparrow\rangle \rightarrow|1 \downarrow\rangle$ and $|0 \uparrow\rangle \rightarrow|1 \downarrow\rangle$ is about $15 \mathrm{meV}$ for $k_{\|}=0$ and decreases to roughly $6 \mathrm{meV}$ for $k_{\|}=k_{\|}\left(E_{F}\right)$. The splittings are to be compared with the line broadening for both the spinflip and the spin-conserving transitions which is governed by the nonparallel subband dispersion of the first and the second subbands. For high carrier concentrations as present in our sample this mechanism dominates all other sources of line broadening. At $B=0 \mathrm{~T}$ the single particle subband spacings decrease by roughly $35 \mathrm{meV}$ from $k_{\|}=0$ to $k_{\|}=k_{F}=0.04 \AA^{-1}$, accounting for the linewidth of the IST in the single-particle picture. From this analysis we would thus not expect to resolve the four possible resonances.

It is well known from Raman experiments that spinconserving IST are influenced by the depolarization field whereas spin-flip excitations are not [1]. As we interpret line $A$ as a superposition of the spin-flip transitions and line $C$ as a superposition of the spin-conserving resonances, the energetic difference for $B \rightarrow 0 \mathrm{~T}$ between both represents to a good approximation the depolarization shift. This interpretation is further supported by calculating the $k_{\|}$-dependent single particle transition energies which are in very close agreement with the $B \rightarrow$ 0 position of resonance $A$. Moreover, the depolarization field reduces the line broadening of IST as has been predicted theoretically [14] and recently been observed in inelastic light scattering experiments [15]. In our case the depolarization-shifted line $C$ has a full width at half maximum of roughly $8 \mathrm{meV}$ which is much narrower than expected from the $k_{\|}$dependence of the subband spacings. Line $A$, on the other hand, exhibits a pronounced lowenergy shoulder even at low magnetic fields where the spin splitting between both spin-flip transitions is much smaller than the linewidth.

We now address the question how the spin-conserving transitions are excited with in-plane polarized light. Whereas only weak excitations caused by interband coupling in narrow-gap materials [16] are possible at $B=0 \mathrm{~T}$ another excitation mechanism is predominant in the Voigt configuration for $B \neq 0 \mathrm{~T}$. At finite magnetic fields the light wave polarized in the $x$ direction (the "cyclotron resonance active" mode) is no longer transverse [17] but acquires an electric field component in the growth direction. The important fact is that this component $E_{y}^{\text {ind }}$ is proportional to the magnetic field $B$ so that the intensity $I$ of the depolarization shifted IST (A) varies as $I \propto B^{2}$ [8]. As shown in the inset of Fig. 1 this is indeed what we find experimentally. The above reasoning also explains why even in the limit $B \rightarrow 0 \mathrm{~T}$ we observe the full depolarization shift of line $C$.

The excitation mechanism of the spin-flip transitions is of a completely different nature. As has been shown for bulk InSb [18] the band nonparabolicity in narrowgap III-V semiconductors may induce combined spin-flip and cyclotron resonance excitations. Our calculations, however, suggest that for our samples, this mechanism is too weak to account for the observed resonance. Inversion asymmetry induced by external electric fields [19] also leads to the possibility of spin-flip excitations. The symmetry of our structure, however, rules out this mechanism, too. We thus conclude that here the inherent bulk inversion asymmetry is responsible for the spin-flip excitations at high $k_{\|}$values.

The dominant inversion asymmetry terms in the Hamiltonian have the schematic form $G\left(P_{i} P_{j}+P_{j} P_{i}\right)$, where $G$ is a material constant and $P_{i, j}$ the canonical momentum [20]. As these terms have only a small influence on the energy dispersion and, in particular, on the $B$ dependence of the transition energies we may neglect them in the computation of the eigenenergies, but we do include bulk inversion asymmetry in the computation of the eight-component wave function and the Hamiltonian of electron-photon interaction. Since the value of $G$ is not known for InAs, we take $G=3 \hbar^{2} / 2 m_{0}$ as in InSb [20] and assume a level broadening of $\hbar / \tau=5 \mathrm{meV}$. The depolarization effects are quantitatively taken into account by generalizing the approach of Ando [21] for the Voigt configuration.

We obtain the following important results. The transition probability for the spin-flip processes increases strongly as a function of the magnetic field. The matrix elements of the two spin-conserving transitions are roughly equal and about twice as large as for both spinflip transitions at all magnetic fields. Furthermore, all possible excitations are induced by the same radiation component $\alpha_{x}$. The other light component $\alpha_{z}$ can be shown to give only a negligibly small contribution to the absorption. This prediction has been confirmed in an additional experiment with predominantly $x$-polarized light where the relative transmission for both types of excitations is significantly enhanced as compared to $z$-polarized light.

For the above reason we show in the calculated spectrum of Fig. 3 only results for the polarization $\alpha_{x}$. In agreement with the experiment two peaks appear, although we deal with four transitions. The resonance at $\hbar \omega=135 \mathrm{meV}$ represents the superposition of the two depolarization shifted spin-conserving transitions with almost equal transition energies and oscillator strengths. Their resonance width is drastically reduced by the depolarization field and not determined by the nonparabolicity broadening. Also, the resonance position is almost field independent as the depolarization shift decreases with increasing magnetic field. The broader peak 


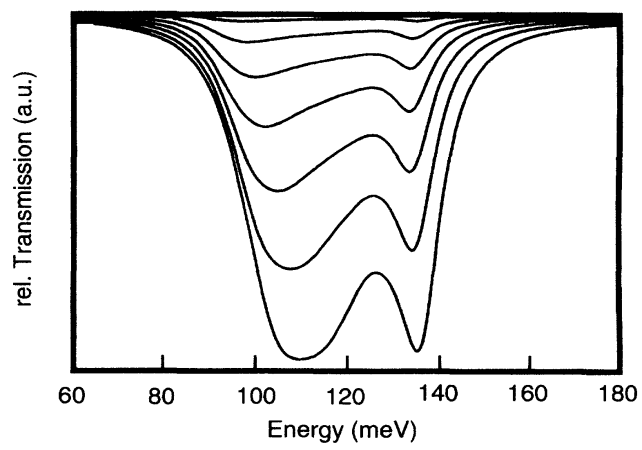

FIG. 3. Calculated transmission spectrum of the spinconserving and spin-flip transitions for light polarization $\alpha_{x}$. The calculation is carried out for magnetic fields between $B=2 \mathrm{~T}$ and $B=14 \mathrm{~T}$ with $\Delta B=2 \mathrm{~T}$. Quantitative agreement is reached between our theoretical description and the experimental data.

at lower energies results from a superposition of both spin-flip transitions which are not resolved because of the nonparabolicity-induced broadening and the reduced spin splitting at high values of $k_{\|}$. Both spin-flip transitions contribute with roughly the same oscillator strength to the absorption since the matrix elements are very similar and the occupation of the spin-split subbands plays only a minor role because $E_{\text {splitting }} \ll E_{F}$. In the final description the strengths of the spin-conserving and spin-flip transitions are almost the same as a result of the interplay between the matrix elements and the depolarization integrals. Hence, the very good agreement between the results of our calculation and the experimental observation gives strong support for the proposed mechanisms.

To summarize, we have observed intersubband optical transitions in the Voigt geometry in InAs/AlSb quantum wells. We demonstrate that spin-conserving transitions can be induced by an in-plane magnetic field although the incident light is polarized in the plane of the two-dimensional electron gas. Furthermore, the joint action of bulk inversion asymmetry and an in-plane magnetic field are quantitatively identified to make a direct observation of intersubband excitations involving a spin-flip possible. The simultaneous observation of spin-flip and spin-conserving transitions allows us to measure directly the depolarization shift in an infrared transmission experiment. Moreover, our results confirm the prediction that the depolarization effect strongly reduces the broadening of intersubband resonances resulting from conduction band nonparabolicity.
The work in Munich was sponsored by the Volkswagen Stiftung. The Santa Barbara group gratefully acknowledges support from the Office of Naval Research and from QUEST, the NSF Science and Technology Center for Quantized Electronic Structures (Grant No. DMR 9120007). The Munich-Santa Barbara cooperation is also supported by a joint NSF/European grant (Grant No. ECUS 015:9826).

*Permanent address: Institute of Physics, Polish Academy of Science, 02668 Warsaw, Poland.

[1] A. Pinczuk and G. Abstreiter, in Light Scattering in Solids $V$, edited by $M$. Cardona and G. Güntherodt (SpringerVerlag, Berlin, 1989), p. 153.

[2] W. Beinvogl and J.F. Koch, Phys. Rev. Lett. 40, 1737 (1978).

[3] E. Batke, G. Weimann, and W. Schlapp, Phys. Rev. B 43, 6812 (1991).

[4] A.D. Wieck, E. Batke, D. Heitmann, J.P. Kotthaus, and E. Bangert, Phys. Rev. Lett. 53, 493 (1984).

[5] E. O. Kane, J. Phys. Chem. Solids 1, 249 (1957).

[6] Y.F. Chen, M. Dobrowolska, J. K. Furdyna, and S. Rodriguez, Phys. Rev. B 32, 890 (1985).

[7] G. Lommer, F. Malcher, and U. Rössler, Phys. Rev. Lett. 60, 728 (1988).

[8] W. Zawadzki, Semicond. Sci. Technol. 2, 550 (1987).

[9] T. Ando, A. B. Fowler, and F. Stern, Rev. Mod. Phys. 54, 473 (1982)

[10] A.D. Wieck, E. Batke, D. Heitmann, and J. P. Kotthaus, Phys. Rev. B 30, 4653 (1984).

[11] G. Tuttle, H. Kroemer, and J.H. English, J. Appl. Phys. 65, 5239 (1989).

[12] C. Nguyen, B. Brar, H. Kroemer, and J. H. English, Appl. Phys. Lett. 60, 1854 (1992).

[13] W. Zawadzki, S. Klahn, and U. Merkt, Phys. Rev. B 33, 6916 (1986)

[14] M. Zaluzny, Phys. Rev. B 43, 4511 (1991).

[15] G. Brozak, B. V. Shanabrook, D. Gammon, D. A. Broido, R. Beresford, and W. I. Wang, Phys. Rev. B 45, 11399 (1992).

[16] W. Zawadzki, J. Phys. C 16, 229 (1983).

[17] W. Zawadzki, Adv. Phys. 23, 435 (1974).

[18] B. D. McCombe, Phys. Rev. 181, 1206 (1969).

[19] E. I. Rashba and V.I. Sheka, Fiz. Tverd. Tela 3, 1735 (1961) [Sov. Phys. Solid State 3, 1257 (1961)]; Fiz. Tverd. Tela 3, 1863 (1961) [Sov. Phys. Solid State 3, 1357 (1961)].

[20] E. O. Kane, in Narrow Gap Semiconductors. Physics and Applications, edited by W. Zawadzki (Springer-Verlag, Berlin, 1980), p. 13.

[21] T. Ando, J. Phys. Soc. Jpn. 44, 475 (1978). 